\title{
New name and new perspectives for the International Journal of Public Health, formerly SPM
}

\author{
Thomas Abel, Alfredo Morabia, T. Kohlmann
}

\section{Dear Readers!}

In the year 2001 Social and Preventive Medicine (SPM) came with a new cover, changes in its layout and a new subtitle (International Journal of Public Health). More importantly, however, the journal had revised its editorial board, content, structure and style. Aims and scope continued to include original works from many disciplines and topic areas in Public Health. Yet, within this broad perspective the new SPM has put more emphasis on research based on Health Surveys, Risk Factor Surveillance and Health Promotion. SPM has provided a scientific forum to publish findings from research studies in these areas, encouraged international comparisons, supported and stimulated the debate on methodology and ethics.

Since 2001, SPM started new sections dedicated to the international comparison of health determinants and to the history of epidemiologic methods, to hints and kinks in survey methodology and to public health in Eastern Europe. Today, we draw a positive balance of these six years. Three major changes in the format of the journal and its "human resources" are relevant for the future development and we use this editorial to inform the readership and potential authors of these changes.

First: after a transition during which it had two names, the journal is now going to be the International Journal of Public Health (Int J Public Health). This change is meant to more clearly signify its interdisciplinary contributions from and across the different Public Health theories, methods and paradigms.

Second: language has always been a key issue for our progress and in our publication policy. We have published papers in three major traditional research languages, English, German and French. This has allowed researchers from those language backgrounds to communicate their knowledge and findings in their mother tongue, which - when it comes to substantive text not highly formalizedcan be of considerable advantage to the quality of the paper. However, we also recognize the need for Public Health knowledge transfer across languages. Thus, the International Journal of Public Health will work towards new models of publishing that promote the scientific exchange across cultures and help to reduce publication language bias. We admit that we don't have the solution yet.

Third: Alfredo Morabia has taken the editorship of another journal (Preventive Medicine) and is leaving the position as one of the two editors-in-chief. He will be replaced by Thomas Kohlmann who has been associated editor of SPM since 2001. Thomas Kohlmann is a well known expert in empirical methods. His expertise covers a broad range of topical areas featuring quality of life, epidemiology of pain an social inequalities.

Sustainable success of the Journal depends on several factors, but principally on the quality of the work it attracts the expertise of its associate editors, their ability to find the appropriate reviewers, and the dedication of the reviewers, an often unsung but essential component of the peer-review. We will build on this team work and spirit in our contribution to the Health of the international public.

\section{On behalf of the Editorial Board \\ The Editors-in-Chief}

Thomas Abel, Alfredo Morabia and Thomas Kohlmann

\section{On to new shores ...}

Alfredo Morabia is leaving Switzerland and moving West to take on new professional challenges at the City University of New York. He is leaving as our co-editor in chief and has become new editor in chief of Preventive Medicine. Yet he will continue his work for our journal as member of the editorial board.

The evolution from SPM into IJPH has in many ways been tied to Alfredo's dedication and inspiration. His innovative thinking, his enthusiasm combined with hard work have helped SPM to develop as an international journal that is today recognised by the Public Health community world wide. We will continue on that road but will surely miss Alfredo as a great colleague and wonderful companion. Thank you, Alfredo and the best of luck for the future.

On behalf of the Editorial Board and the Editorial Team Thomas Abel 